\title{
Highly Efficient Molecular Ionization Probed by Few-cycle Laser Pulses
}

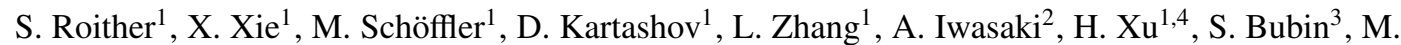
Atkinson $^{3}$, K. Varga ${ }^{3}$, K. Yamanouchi ${ }^{2}$, A. Baltuška ${ }^{1}$, and M. Kitzler ${ }^{1, a}$

1 Photonics Institute, Vienna University of Technology, Vienna, Austria

2 Department of Chemistry, School of Science, The University of Tokyo, Tokyo, Japan

3 Department of Physics and Astronomy, Vanderbilt University, Nashville, Tennessee, USA

4 State Key Laboratory on Integrated Optoelectronics, Jilin University, Changchun, China

\begin{abstract}
Field ionization of hydrocarbon molecules to high charge states is studied as a function of laser pulse duration, peak intensity and molecular alignment. Results are in agreement with the recently proposed mechanism of multi-bond enhanced ionization.
\end{abstract}

Molecules subject to strong laser pulses may become ionized during their interaction with the laser field. The details of the accompanying field driven internal electronic dynamics are still far from being understood - in particular for the case of polyatomic molecules, e.g. [1]. After the removal of electrons not only the charge density will redistribute very quickly within the molecule $[2,3]$, also the molecule itself may undergo partly severe structural deformation. Eventually the multiply charged complex may break into two or several charged fragments and the excessive molecular potential energy is released into kinetic energy of the resulting set of final fragment ions.

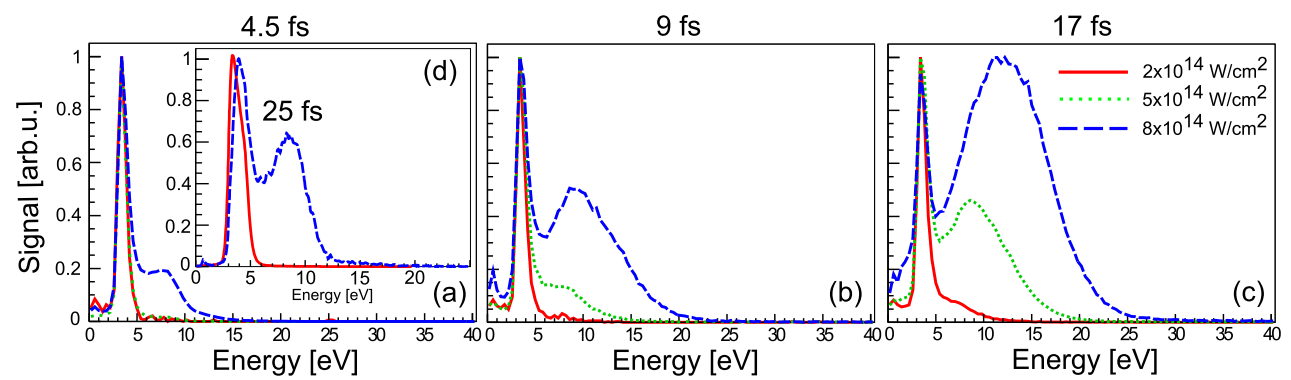

Fig. 1. Measured energy spectra of protons created by the fragmentation of $\mathrm{C}_{2} \mathrm{H}_{4}$ molecules following the interaction with $4.5 \mathrm{fs}$ (a), 9 fs (b), $17 \mathrm{fs}$ (c), long (FWHM) laser pulses with peak intensities (W/cm ${ }^{2}$ ) $2 \times 10^{14}$ (full lines), $5 \times 10^{14}$ (dotted lines), $8 \times 10^{14}$ (dashed lines). (d) Energy spectra of protons ejected from the doubly (full line) and triply (dashed line) charged ion during two- and three-body fragmentations, respectively.

Recently it was found that the interaction of hydrocarbon molecules with $\approx 25$ fs laser pulses at moderate intensities of a few $10^{14} \mathrm{~W} / \mathrm{cm}^{2}$ results in complete molecular fragmentation and the emission of protons with considerable kinetic energy [4]. Using multi-particle coincidence analysis it was shown that the reason for the observed highly energetic protons is that the molecules reach unexpectedly high charge states (in excess of $10+$ for $\mathrm{C}_{4} \mathrm{H}_{6}$ ) during the laser pulse. Based on experimental evidence it was suggested that a novel type of molecular ionization, i.e., a multi-bond version of the

a e-mail: markus.kitzler@tuwien.ac.at

This is an Open Access article distributed under the terms of the Creative Commons Attribution License 2.0, which permits unrestricted use, distribution, and reproduction in any medium, provided the original work is properly cited. 

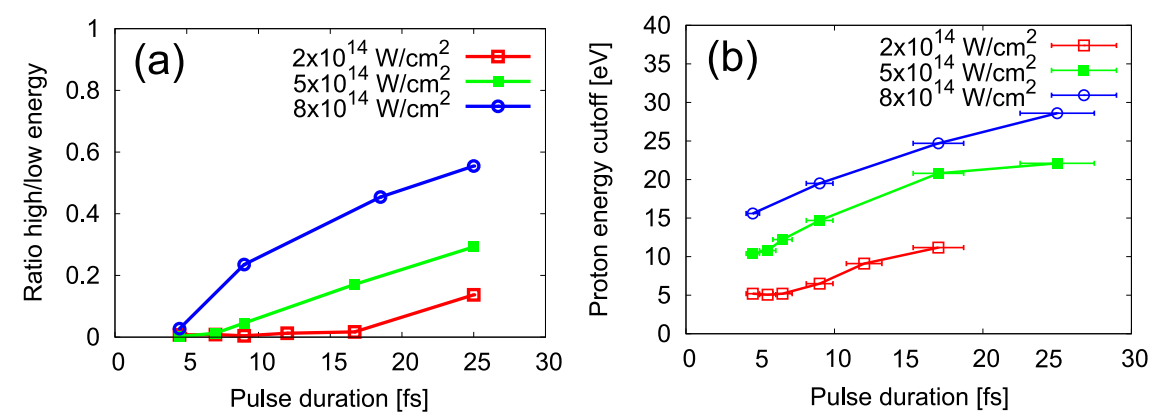

Fig. 2. (a) Ratios of the number of protons ejected from $\mathrm{C}_{2} \mathrm{H}_{4}$ with energies exceeding $12 \mathrm{eV}$ to the number of all detected protons as a function of laser pulse duration for the three intensities $2 \times 10^{14} \mathrm{~W} / \mathrm{cm}^{2}$ (empty squares), $5 \times 10^{14} \mathrm{~W} / \mathrm{cm}^{2}$ (full squares), $8 \times 10^{14} \mathrm{~W} / \mathrm{cm}^{2}$ (empty circles). (b) Cut-off values of proton spectra, as defined in [4], as a function of pulse duration, shown for the same three intensities and with the same symbols as in (a). The lines are only to guide the eye.

well known enhanced-ionization (EI) mechanism [5, 6], is responsible for the surprising ionization behavior.

In this submission we report on experiments that test this explanation. The experiments are based on the fact that the EI mechanism necessitates a certain amount of time, as this mechanism involves coupled fast electronic and slower nuclear motion. The determining nuclear motion for this mechanism in the case of hydrocarbons is the very fast stretching of the $\mathrm{C}-\mathrm{H}$ bonds, which is expected to take place on a time-scale of $10 \mathrm{fs}$, to the critical internuclear distance at which EI occurs [5, 6]. In our experiments we therefore use 4.5 fs laser pulses generated by spectral broadening of laser pulses from a Titanium-Sapphire laser amplifier system and temporal recompression using chirped mirrors. The pulses are directed into a COLTRIMS apparatus, where they are focused onto a cold supersonic jet of ethylene, $\mathrm{C}_{2} \mathrm{H}_{4}$, and acetylene, $\mathrm{C}_{2} \mathrm{H}_{2}$, molecules. The three-dimensional momenta of the resulting ionic fragments from a single molecule are recorded as described previously [4].

Measured proton spectra for ethylene are shown in Fig. 1 for three exemplary pulse durations ( $4.5 \mathrm{fs}, 9 \mathrm{fs}, 17 \mathrm{fs}$ ) and three pulse peak intensities. Clearly, for a given intensity the proton energy spectra extend to higher energies with increasing pulse duration, see Fig. 1. While for the shortest pulse duration of $4.5 \mathrm{fs}$ [Fig. 1(a)] the proton energies do not significantly exceed $10 \mathrm{eV}$ even for the highest intensity of $8 \times 10^{14} \mathrm{~W} / \mathrm{cm}^{2}$ (dashed lines), the proton energies extend up to $25 \mathrm{eV}$ when the pulse duration is increased to $17 \mathrm{fs}$ [Fig. 1(c)].

The cut-off energy of the proton spectra can be related to the mean charge state of the ethylene ions before fragmentation [4]. Using coincidence selection we extract the proton energy spectra created by Coulomb explosion of doubly and triply charged molecular ions during two- and three-body fragmentations [Fig. 1(d)]. By comparing these spectra to those measured for the $4.5 \mathrm{fs}$ pulse in Fig. 1(a) it becomes evident that even at $8 \times 10^{14} \mathrm{~W} / \mathrm{cm}^{2}$ they are created by fragmentations from low charge states. As the pulse duration is increased to 9 fs and further to $17 \mathrm{fs}$ [Figs. 1(b) and (c)] both the proton energy cut-off and the number of protons that reach higher energies increase. We can consider the results for the $17 \mathrm{fs}$ pulse as those of the long-pulse regime [4]. Indeed, the proton spectrum measured with $8 \times 10^{14} \mathrm{~W} / \mathrm{cm}^{2}$ at this pulse duration shows a pronounced high-energy part that extends to similar energies in excess of $20 \mathrm{eV}$ as measured previously [4]. For the smaller intensities the molecules are only doubly or triply ionized, as we can see by comparing the proton energies to those of doubly and triply ionized molecules shown in Fig. 1(d).

To understand the dependence of the ionization behavior on the laser peak intensity we plot the number of protons with energies in excess of $12 \mathrm{eV}$ normalized to the total number of protons for the three different intensities as a function of pulse duration in Fig. 2(a). The border of $12 \mathrm{eV}$ corresponds to the energy that protons reach when they are created during complete fragmentation of the ethylene molecule from charge states higher or equal of +6 [4]. Fig. 2(a) thus allows to follow the onset and evolution of a complete molecular fragmentation as a function of time and intensity. A very similar information can be taken from the cut-off value of the proton spectra, defined in [4], shown in Fig. 2(b). 


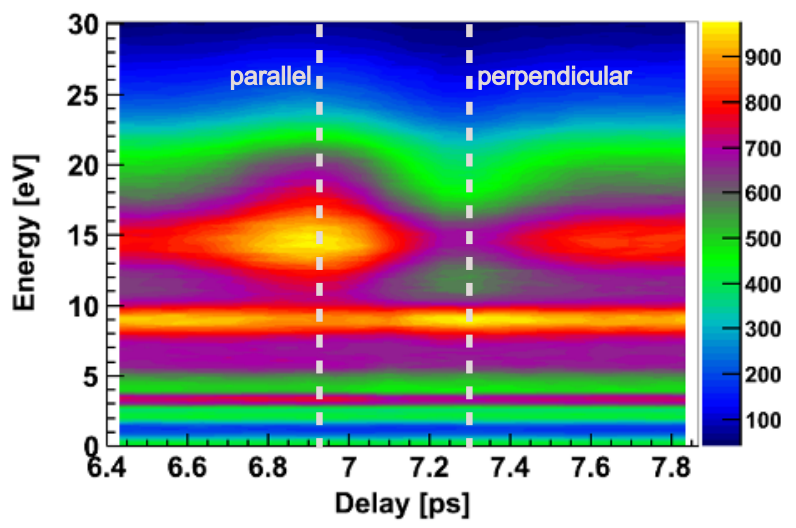

Fig. 3. Energy spectra of protons ejected from acetylene molecules as a function of the time delay between the $\approx 50 \mathrm{fs}$ impulsive alignment pulse and the $4.5 \mathrm{fs}$ ionizing pulse. The molecular axis is aligned parallel to the polarization axis of the linearly polarized ionizing pulse with peak intensity of $7 \times 10^{14} \mathrm{~W} / \mathrm{cm} 2$ around $6.9 \mathrm{ps}$ and perpendicular around $7.3 \mathrm{ps}$.

Fig. 2(a) shows that for pulse durations shorter than $10 \mathrm{fs}$ complete fragmentation of the ethylene molecule is negligible even for the highest intensity. For long pulses around $20 \mathrm{fs}$ complete molecular fragmentations are observed even for intensities as low as $2 \times 10^{14} \mathrm{~W} / \mathrm{cm}^{2}$, and at $8 \times 10^{14} \mathrm{~W} / \mathrm{cm}^{2}$ more than half of all detected molecules are completely fragmented. These data confirm the proposed multibond EI mechanism [4]. Additionally, they provide new insight into the intensity dependence of the ionization-fragmentation mechanism in a thus far unexplored temporal regime.

As ionization of molecules critically depends on their electronic orbital structure along the electron emission direction, an interesting question in the multi-bond EI mechanism is its dependence on the angle of the molecular axis to the polarization direction of a linearly polarized ionizing pulse. To investigate this dependence we performed experiments on aligned acetylene molecules using impulsive alignment by a preceding stretched $\approx 50 \mathrm{fs}$ long pulse. Fig. 3 shows that the proton energy spectra sensitively depend on the molecular alignment. Maximum probability of generating high charge states and therewith high proton energies is found for parallel alignment.

In conclusion, we present experimental data that confirm the multi-bond enhanced-ionization mechanism of polyatomic molecules that was proposed previously [4]. Additionally, we investigated the dependence of this mechanism on the molecular alignment.

\section{References}

1. M. Lezius, V. Blanchet, M. Y. Ivanov, and A. Stolow, J. Chem. Phys. 117, 1575 (2002).

2. F. Remacle and R. D. Levine, PNAS 103, 6793 (2006).

3. J. Breidbach and L. Cederbaum, Phys. Rev. Lett. 94, 033901 (2005).

4. S. Roither et al., Phys. Rev. Lett. 106, 163001 (2011).

5. T. Seideman, M. Ivanov, and P. Corkum, Phys. Rev. Lett. 75, 2819 (1995).

6. T. Zuo and A. D. Bandrauk, Phys. Rev. A 52, R2511 (1995). 